The authors reported no conflicts of interest.

The Journal policy requires editors and reviewers to disclose conflicts of interest and to decline handling or reviewing manuscripts for which they may have a conflict of interest. The editors and reviewers of this article have no conflicts of interest.

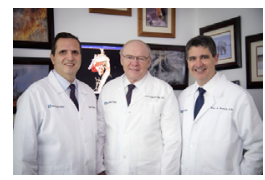

\section{REPLY: NOVEL AORTIC IMAGING MODALITIES: MINE DETECTORS OR JUST METAL DETECTORS Reply to the Editor:}

We agree with Ma and colleagues ${ }^{1}$ about the potential limitations of the finite element analysis and 3-dimensional models derived from computed tomography angiography of the thoracic aorta used by Wang and colleagues ${ }^{2}$ to measure wall stress. Indeed, unlike tricuspid aortic valves, bicuspid valves are associated with different hemodynamic parameters and thoracic aortic wall substrates that can influence wall stress and the associated clinical downstream consequences. In fact, the study by Wang and colleagues ${ }^{2}$ made many assumptions, including identical aortic wall composition and thickness and physiologic pressures among allcomers. Although they used patient-derived imaging data in their modeling, as pointed out by Ma and colleagues, ${ }^{1}$ it lacked validation and clinical correlation. Furthermore, fluid-structure interaction is critical to account for in an analysis of the aorta and the oscillatory forces within it. Nevertheless, it was an important contribution because it rightly challenged the disproportionate importance assigned to aortic diameter when it comes to making recommendations about timing of surgery. Smaller $(<5 \mathrm{~cm})$ thoracic aortas can experience substantial wall stress and this may explain aortic dissections in patients who do not meet the guidelines-based size criteria for intervention.

With regard to biomechanical data derived from in vivo multimodality imaging, ${ }^{1}$ the jury is still out on their specificity and predictive value when it comes to aortic dissection or rupture. Those modalities, although promising, are not yet ready for prime time because they need rigorous evaluation and actual risk calibration. With the expansion of computational power and associated advances in analysis of fluid-structure interaction, rapid modeling based on patient-specific data-including physiologic and anatomic details - is now feasible. As we mentioned before, ${ }^{3}$ the goal of early and more accurate identification of patients who will benefit from preventative aortic replacement using precision medicine techniques will become a reality soon. In the meantime, there is no substitute for good surgical judgment and skill when deciding on the timing of prophylactic aortic surgery.

Faisal G. Bakaeen, MD

Eric E. Roselli, $M D$

Lars G. Svensson, $M D, P h D$

Department of Thoracic and Cardiovascular Surgery

Aorta Center

Heart Vascular and Thoracic Institute

Cleveland Clinic

Cleveland, Ohio

\section{References}

1. Ma W, Liang Y, Wang C. Assessment of wall stress distribution in thoracic ascending aortic aneurysm: simulation, prediction, and prevention. J Thorac Cardiovasc Surg. 2020;160:e101.

2. Wang Z, Flores N, Lum M, Wisneski A, Xuan Y, Inman J, et al. Wall stress analysis in $\geq 5 \mathrm{~cm}$ versus $<5 \mathrm{~cm}$ ascending thoracic aortic aneurysm patients. $J$ Thorac Cardiovasc Surg. February 19, 2020 [Epub ahead of print].

3. Bakaeen FG, Roselli EE, Svensson LG. Commentary: thoracic aortas: more to stress about than just size. J Thorac Cardiovasc Surg. February 21, 2020 [Epub ahead of print].

\section{https://doi.org/10.1016/j.jtcvs.2020.04.109}

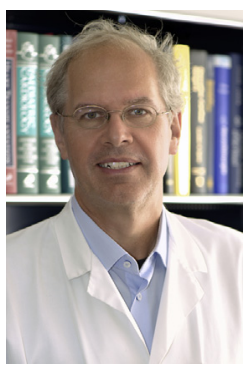

REPLY: ACCURATE EVALUATION OF THE RISK OF ACUTE AORTIC EVENTS: STILL ROOM FOR IMPROVEMENT

\section{Reply to the Editor:}

Computational models to better assess the wall properties of the thoracic aorta and thus the individual risk assessment of a subsequent acute aortic syndrome (eg, intramural hematoma, aortic dissection) would be extremely welcome in daily clinical work. The article by Wang and colleagues, ${ }^{1}$ the comment by Carrel and Schoenhoff, ${ }^{2}$ and the letter to the editor by Ma and colleagues ${ }^{3}$ clearly demonstrate not only the interest in, but also the importance of this field, given that inaccurate prediction may lead to an unexpected higher risk of acute aortic complications.

There is no doubt that size alone is not a good indicator of the risk of dissection in any given patient, because numerous individual risk factors may influence the prediction and interpretation of this particular risk; however, size is considered a classical parameter in the guidelines dealing with indications to replace a dilated aortic segment, whereas several additional risk factors (eg, age, family history of acute aortic disease and connective tissue disease) may provide valuable additional information.

In a previous editorial comment, ${ }^{2}$ we stated that more modern methods than simple imaging, such as computational modeling and biomechanics, may provide important information regarding aneurysm geometry in general and 
The author reported no conflicts of interest.

The Journal policy requires editors and reviewers to disclose conflicts of interest and to decline handling or reviewing manuscripts for which they may have a conflict of interest. The editors and reviewers of this article have no conflicts of interest.

how material properties may affect wall stress. In the view of the present results in this particular field, this might not be powerful enough. Because artificial intelligence is actually a common entity in experimental medicine as well as clinical medicine, such an approach based on dedicated algorithms may be a valuable tool for additional pattern recognition and thus better predictive capabilities to facilitate decision making.

The new technology presented by Wang and colleagues did not allow the detection of close correlations between wall stress and size of the dilated aortic segment, and as a major finding, there was no diameter threshold at which a sharper correlation between wall stress and aneurysm size could be observed. This was perhaps due to the fact that the finite element analysis considered the aortic tissue as a uniform material (omitting the differences in the wall properties between connective tissue diseases and atherosclerotic disease), which most likely is not the case. In combination with additional data generated by echocardiography (a readily reproducible diagnostic tool), as well as by magnetic resonance imaging, this technology may help qualify and quantify the aortic wall properties at different places in the ascending aorta and in the aortic arch, for instance.

Both Wang and colleagues and $\mathrm{Ma}$ and colleagues emphasize that a combination of multimodal imaging and its integration into a computational modeling may be a precise solution for such a complex and heterogeneous clinical situation. For the accurate evaluation of the risk of acute aortic events, there is still room for improvement.

\section{Thierry Carrel, $M D$ Department of Cardiovascular Surgery University Hospital and University of Bern Bern, Switzerland}

\footnotetext{
References

1. Wang Z, Flores N, Lum M, Wisneski AD, Xuan Y, Inman J, et al. Wall stress analyses in $\geq 5 \mathrm{~cm}$ vs $<5 \mathrm{~cm}$ ascending thoracic aortic aneurysm patients. J Thorac Cardiovasc Surg. February 19, 2020 [Epub ahead of print].

2. Carrel T, Schoenhoff F. Diameter alone is not precise enough, but wall stress analysis may facilitate decision-making for indications for prophylactic aortic repair. J Thorac Cardiovasc Surg. February 24, 2020 [Epub ahead of print].

3. Ma W, Liang Y, Wang C. Assessment of wall stress distribution in thoracic ascending aortic aneurysm: simulation, prediction, and prevention. J Thorac Cardiovasc Surg. 2020;160:e101.
}

https://doi.org/10.1016/j.jtcvs.2020.04.146

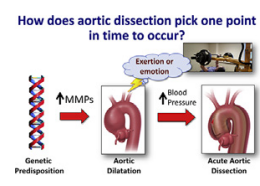

REPLY: IMAGING IS NOT EVERYTHING AS REGARDS THE AORTA: TISSUE STRENGTH AND BLOOD PRESSURE MATTER AS WELL?

Reply to the Editor:

We read with interest the Commentary by Ma and colleagues, ${ }^{1}$ in which they provide their vision for integrating information from 3 imaging modalities into a unified whole for thoracic aortic assessment, prediction, and decision-making. We find their schematic (Central Image in Ma and colleagues ${ }^{1}$ ) demonstrating integration of the modalities of echocardiography (providing stiffness information), computed tomography scanning (providing strain information), and sheer stress calculation toward achieving a unified analysis for prediction of aortic behavior and clinical decision-making insightful and cogent. We especially applaud their attention to speckle-tracking capability via echocardiography, the potential of which is underused. ${ }^{2}$ Speckle tracking uses the naturally occurring aortic tissue speckle patterns under ultrasound to track expansion between systole and diastole.

We have 4 comments to make to augment their analysis and put it into perspective:

1. Any engineering analysis of the potential for dissection or rupture of the aorta is incomplete without consideration of the ultimate tensile strength of the aorta. Currently, this cannot be assessed by purely radiographic means, but rather requires actual tensile testing of the aorta in a "stretching" apparatus. We and others have reported extensively on such empirical values of tensile strength of the human thoracic aorta in normal and diseased states. $^{3-5}$ This information needs to be incorporated into any predictive algorithm.

2. Any engineering analysis of the potential for dissection or rupture of the aorta is incomplete without consideration of the ambient blood pressure. Ambient blood pressure is the agent that actually stretches and jeopardizes the aortic wall in the clinical setting. Our clinical studies have shown that not only baseline blood pressure but also exacerbations during emotional stress or strenuous exercise play a critical role in inducing aortic dissection $^{6,7}$ (Figure 1).

3. It is important that our readership, whose physics studies may have been completed many years ago, recognize the difference between wall stress and shear stress. Wall stress in the aorta represents the force trying to pull the aortic wall apart, but shear stress represents the interaction between the flowing blood and the intima of the aorta (the "friction" or "drag" between flowing blood and the arterial wall, so to speak). Wall stress is the classically, mechanically important parameter. Shear stress 